\title{
Semileptonic and Leptonic Charm Decays at BESIII
}

\section{Sanqiang Qu*}

School of Physics, Nankai Unibersity, Tianjin, P.R.China,

E-mail: qusq@mail.nankai.edu.cn

(On behalf of the BESIII collaboration)

BESIII has collected $e^{+} e^{-}$collision data samples corresponding to luminosities of $2.93 \mathrm{fb}^{-1}$ and $3.19 \mathrm{fb}^{-1}$ at center-of-mass energies of 3.773 and $4.178 \mathrm{GeV}$, respectively. In addition, data sample with a luminosity of about $3 \mathrm{fb}^{-1}$ at centerof-mass energies between 4.19 and $4.23 \mathrm{GeV}$ are used to study $D_{s}$ decay. We report recent measurements that include the decays $D_{(s)}^{+} \rightarrow l^{+} v(l=\mu, \tau), D^{0} \rightarrow$ $K^{-} \mu^{+} v_{\mu}, D^{+} \rightarrow \eta / \omega \mu^{+} v_{\mu}, D^{0(+)} \rightarrow \pi \pi e^{+} v_{e}, D^{+} \rightarrow K_{1}(1270)^{0} e^{+} v_{e}, D_{s}^{+} \rightarrow$ $\eta^{(\prime)} e^{+} v_{e}$ and $D_{s}^{+} \rightarrow K^{(*)} e^{+} v_{e}$. From these analyses, the decay constants $f_{D_{(s)}}$, the semileptonic form factors $f_{+}^{P}(0)\left(P=K, \quad \eta^{(\prime)}\right)$ and the CKM matrix elements $\left|V_{c d(s)}\right|$ are determined precisely. These results can verify the LQCD calculations of $f_{D_{(s)}}, f_{+}^{P}(0)$ and the CKM matrix unitarity. Precision tests of lepton-flavor universality are also made using data of these decays. 


\section{Introduction}

Semileptonic and leptonic decays of the ground-state charm mesons are well described in the Standard Model (SM). In these decays, the strong and weak interactions can be well separated, as shown in Fig. 1.

In the purely leptonic decay of the charmed meson $D_{(s)}^{+}$, the quark and antiquark annihilate into a pair of charged and neutral leptons via a virtual $W$ boson. To the lowest order, the decay rate is given in a simple form:

$$
\Gamma\left(D_{(s)}^{+} \rightarrow l^{+} v\right)=\frac{G_{F}^{2}}{8 \pi} f_{D_{(s)}^{+}}^{2} m_{l}^{2} m_{D_{(s)}^{+}}^{2}\left(1-\frac{m_{l}^{2}}{m_{D_{(s)}^{+}}^{2}}\right)^{2}\left|V_{c d(s)}\right|^{2},
$$

where $G_{F}$ is the Fermi coupling constant, $f_{D_{(s)}^{+}}$is $D_{(s)}^{+}$meson decay constant, $m_{l}$ and $m_{D_{(s)}^{+}}$are the lepton and $D_{(s)}^{+}$masses, $\left|V_{c d(s)}\right|$ is the $c \rightarrow d(s)$ Cabibbo-Kobayashi-Maskawa (CKM) matrix element.

For the semileptonic $D_{(s)}$ decays, the differential decay rate, which can be parameterized by CKM quark mixing matrix and hadronic form factor $f_{+}^{P}\left(q^{2}\right)$, can be simply written as:

$$
\frac{d \Gamma\left(\mathrm{D}_{(\mathrm{s})} \rightarrow \mathrm{Pl} v\right)}{d q^{2}}=\frac{G_{F}^{2}}{24 \pi^{3}}\left|V_{c d(s)}\right|^{2} p_{P}^{3}\left|f_{+}^{P}\left(q^{2}\right)\right|^{2},
$$

where $p_{P}^{3}$ is the meson momentum in the $D_{(s)}$ rest frame, $f_{+}^{P}\left(q^{2}\right)$ is the form factor of hadronic weak current depending on the square of the transferred four momentum $q=p_{D_{(s)}}-p_{P}$.

According to Eq. (1) and Eq. (2), measuring $B\left(D_{(s)} \rightarrow l v\right)$ and $B\left(D_{(s)} \rightarrow P l v\right)$ allows determination of the product $f_{D_{(s)}^{+}}^{2}\left|V_{c d(s)}\right|^{2}$ and $f_{+}^{P}\left(q^{2}\right)\left|V_{c d(s)}\right|^{2}$. Based on these results, we can extract decay constant $f_{D_{(s)}^{+}}$or hadronic form factor $f_{+}^{P}\left(q^{2}\right)$ by using the experimentally measured $\left|V_{c d(s)}\right|$ to test the lattice quantum chromo-dynamics (LQCD) prediction. Reversely, we can also obtain CKM matrix elements $\left|V_{c d(s)}\right|$ by using the predicted value of $f_{D_{(s)}^{+}}$or $f_{+}^{P}\left(q^{2}\right)$ from LQCD to test unitarity of CKM matrix. In addition, the test of the ratio of decay rates of different flavor lepton, which is called lepton flavor universality (LFU), is important to check SM prediction. The study of Semileptonic and leptonic decays can allow us to test the LFU predicted by SM at a high precision.
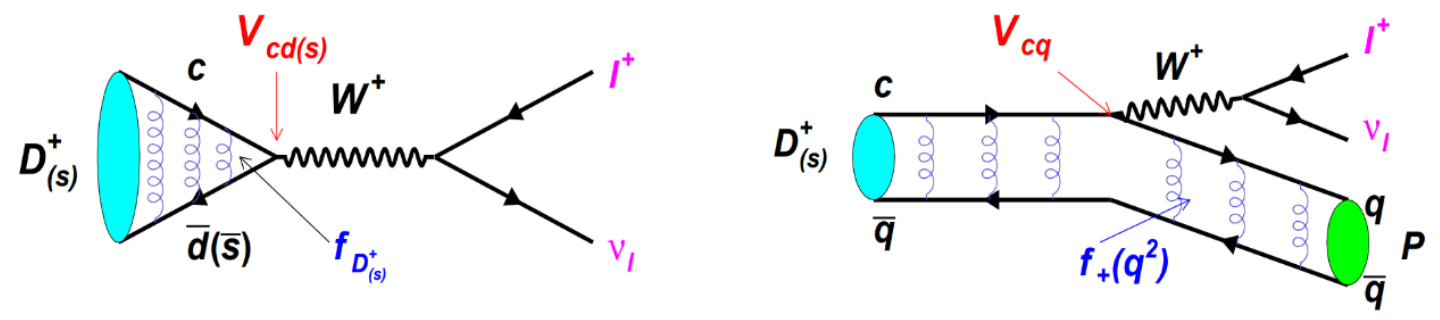

Figure 1: Feynman diagrams for purely leptonic (left) and semileptonic (right) $D_{(s)}^{+}$decays. 


\section{2. $D_{(s)}^{+}$Leptonic decay}

\section{1 $D_{s}^{+} \rightarrow \mu^{+} v_{\mu}$}

Recently, an improved measurement of $D_{s}^{+} \rightarrow \mu^{+} v_{\mu}$ has been reported at BESIII [1]. Using the $3.19 \mathrm{fb}^{-1}$ data sample taken at $\sqrt{s}=4.178 \mathrm{GeV}, B\left(D_{S}^{+} \rightarrow \mu^{+} v_{\mu}\right)$ has determined to be $(5.49 \pm 0.16 \pm 0.15 \pm) \times 10^{-3}$. Figure 2 shows the fit of $M_{m i s s}^{2}$ distributions. Combining our results with the mass of the $D_{s}^{+}$and $\mu^{+}$and the lifetime of the $D_{S}^{+}[2]$, we determine $f_{D_{S}^{+}}\left|V_{c S}\right|=$ $246.2 \pm 3.6 \pm 3.5 \mathrm{MeV}$. Using $\left|V_{c S}\right|$ from a global SM fit [2] or $f_{D_{S}^{+}}$calculated by LQCD [3, 4], we obtain the $f_{D_{S}^{+}}=252.9 \pm 3.7 \pm 3.6 \mathrm{MeV}$ and $\left|V_{c S}\right|=0.985 \pm 0.014 \pm 0.014$. These values are each the most precise results to date. Based on our results, we find that the ratio of $B\left(D_{S}^{+} \rightarrow\right.$ $\left.\mu^{+} v_{\mu}\right)$ over the PDG value [2] of $B\left(D_{s}^{+} \rightarrow \tau^{+} v_{\tau}\right)=(5.48 \pm 0.23) \times 10^{-2}$ is determined to be $9.98 \pm 0.52$, which agrees with the SM prediction within uncertainty.

$2.2 D^{+} \rightarrow \tau^{+} v_{\tau}$

BESIII has also reported the first observation of $D^{+} \rightarrow \tau^{+} v_{\tau}$ with a significance of 5.1 $\sigma$ [5]. By analyzing $2.93 \mathrm{fb}^{-1}$ data taken at the center mass energy of $3.773 \mathrm{GeV}$, the branching fraction (BF) of $D^{+} \rightarrow \tau^{+} v_{\tau}$ has been measured to be $B\left(D^{+} \rightarrow \tau^{+} v_{\tau}\right)=(1.20 \pm 0.24 \pm 0.12) \times 10^{-3}$. Figure 2 shows the fit of $M_{\text {miss }}^{2}$ distributions. Taking the world average $B\left(D^{+} \rightarrow \mu^{+} v_{\mu}\right)=$ $(3.74 \pm 0.17) \times 10^{-4}$, we obtain $R_{\tau / \mu}=\Gamma\left(D^{+} \rightarrow \tau^{+} v_{\tau}\right) / \Gamma\left(D^{+} \rightarrow \mu^{+} v_{\mu}\right)=3.21 \pm 0.64 \pm$ 0.43, which is consistent with the SM expectation of LFU.
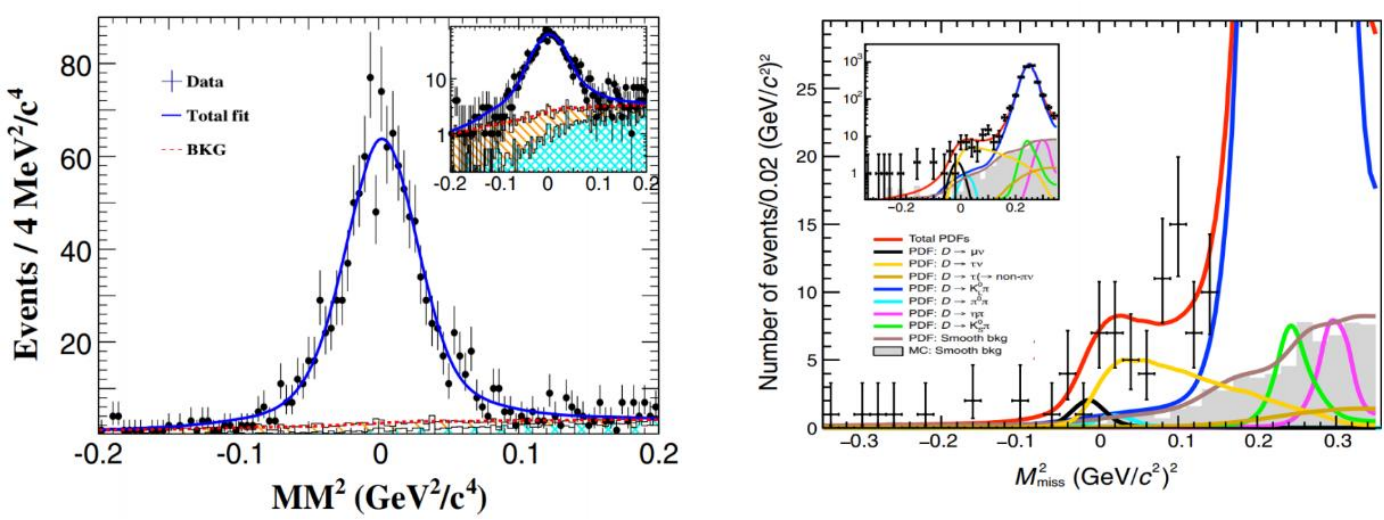

Figure 2: Fit to the distribution of $D_{S}^{+} \rightarrow \mu^{+} v_{\mu}$ (left) and $D^{+} \rightarrow \tau^{+} v_{\tau}$ (right) decays.

\section{3. $D_{(s)}$ Semileptonic decay}

\section{1 $D^{0(+)}$ Semileptonic decay}

3.1.1 $D^{0} \rightarrow K^{-} \mu^{+} v_{\mu}$

Using the same data sample as $D^{+} \rightarrow \tau^{+} v_{\tau}$, BESIII measured the absolute BF of $D^{0} \rightarrow$ $K^{-} \mu^{+} v_{\mu}$ with significantly improved precision: $B\left(D^{0} \rightarrow K^{-} \mu^{+} v_{\mu}\right)=(3.413 \pm 0.019 \pm$ $0.035) \times 10^{-2}$ [6]. Combining with our previous measurement of $B\left(D^{0} \rightarrow K^{-} e^{+} v_{\mu}\right)=$ 
$(3.505 \pm 0.014 \pm 0.033) \times 10^{-2}$ [7], the ratio of the two absoulte BFs is determined to be $R_{\mu / e}=B\left(D^{0} \rightarrow K^{-} \mu^{+} v_{\mu}\right) / B\left(D^{0} \rightarrow K^{-} e^{+} v_{\mu}\right)=0.974 \pm 0.007 \pm 0.012$, which agrees with the theoretical expectation of lepton flavor universality within the uncertainty. Taking input from global fit in the SM [2] and LQCD [8] separately, we determine $f_{+}^{K}(0)=0.7372 \pm 0.0039 \pm$ 0.0030 and $\left|V_{c S}\right|=0.955 \pm 0.005 \pm 0.004 \pm 0.024$.

3.1.2 $D^{+} \rightarrow \eta \mu^{+} v_{\mu}$

The absolute BF of $D^{+} \rightarrow \eta \mu^{+} v_{\mu}$ is measured for the first time [9]. The BF of $D^{+} \rightarrow \eta \mu^{+} v_{\mu}$ is determined to be $B\left(D^{+} \rightarrow \eta \mu^{+} v_{\mu}\right)=(10.4 \pm 1.0 \pm 0.5) \times 10^{-4}$. By studying the differential decay rates in five four-momentum transfer intervals, we obtain the product of the hadronic form factor $f_{+}^{\eta}(0)$ and CKM matrix element $\left|V_{c d}\right|$ to be $f_{+}^{\eta}(0)\left|V_{c d}\right|=0.087 \pm 0.008 \pm 0.002$. Taking the input of $\left|V_{c d}\right|$ from global fit in the SM and $f_{+}^{\eta}(0)$ calculated in theory, we obtain $f_{+}^{\eta}(0)=0.39 \pm 0.04 \pm 0.01$ and $\left|V_{c d}\right|=0.242 \pm 0.022 \pm 0.006 \pm 0.033$. The ratio, $B\left(D^{+} \rightarrow \eta \mu^{+} v_{\mu}\right) / B\left(D^{+} \rightarrow \eta e^{+} v_{e}\right)$, is also determined, obtaining value $0.91 \pm 0.13$, which is consistent with theoretical expectation of LFU.

3.1.3 $D \rightarrow \pi \pi e^{+} v_{e}$

We present an analysis of the decays $D^{0} \rightarrow \pi^{+} \pi^{0} e^{+} v_{e}$ and $D^{+} \rightarrow \pi^{+} \pi^{-} e^{+} v_{e}$ [10]. By performing a partial wave analysis, the $\pi^{+} \pi^{-} S$-wave contribution to $D^{+} \rightarrow \pi^{+} \pi^{-} e^{+} v_{e}$ is observed to be $(25.7 \pm 1.6 \pm 1.1) \times 10^{-2}$ with a statistical significance greater than $10 \sigma$, besides the dominant $P$-wave contribution. This is the first observation of the $S$-wave contribution. We measure the $\mathrm{BFs} B\left(D^{0} \rightarrow \rho^{-} e^{+} v_{e}\right)=(1.445 \pm 0.058 \pm 0.039) \times 10^{-3}, B\left(D^{0} \rightarrow \rho^{0} e^{+} v_{e}\right)=$ $(1.860 \pm 0.070 \pm 0.061) \times 10^{-3}$ and $B\left(D^{0} \rightarrow f_{0}(500) e^{+} v_{e}, f_{0}(500) \rightarrow \pi^{+} \pi^{-}\right)=(6.30 \pm$ $0.43 \pm 0.32) \times 10^{-4}$. An upper limit of $B\left(D^{+} \rightarrow f_{0}(980) e^{+} v_{e}, f_{0}(980) \rightarrow \pi^{+} \pi^{-}\right)<2.8 \times$ $10^{-5}$ is set at the $90 \%$ confidence level. We also calculate the ratio of these BFs $R=$ $\frac{B\left(D^{+} \rightarrow f_{0}(980) e^{+} v_{e}\right)+B\left(D^{0} \rightarrow f_{0}(500) e^{+} v_{e}\right)}{B\left(D^{+} \rightarrow a_{0}(980) e^{+} v_{e}\right)}>2.7$, which is agree with the tetraquark description of the scalar mesons.

3.1.4 $D^{+} \rightarrow K_{1}(1270) e^{+} v_{e}$

We report the first observation of the semileptonic $D^{+}$transition into the axial-vector meson $D^{+} \rightarrow \bar{K}_{1}(1270)^{0} e^{+} v_{e}$ with a statistical significance greater than $10 \sigma$ [11]. The absolute BF of $D^{+} \rightarrow \bar{K}_{1}(1270)^{0} e^{+} v_{e}$ is determined to be $B\left(D^{+} \rightarrow \bar{K}_{1}(1270)^{0} e^{+} v_{e}\right)=\left(2.30 \pm 0.26_{-0.21}^{+0.18} \pm\right.$ $0.25) \times 10^{-3}$. Our result is consistent with the covariant light-front quark model [12] and lightcone QCD sum rules [13] predictions using $\theta_{K_{1}} \approx 33^{\circ}$ or $57^{\circ}$ [12], and clearly rules out the predictions with $\theta_{K_{1}}$ negative [13].

3.1.5 $D^{+} \rightarrow \omega \mu^{+} v_{\mu}$

The absolute $\mathrm{BF}$ of $D^{+} \rightarrow \omega \mu^{+} v_{\mu}$ decay is measured to be $B\left(D^{+} \rightarrow \omega \mu^{+} v_{\mu}\right)=(17.7 \pm$ $1.8 \pm 1.1) \times 10^{-4}$ [14]. Its ratio with the world average value of the $\mathrm{BF}$ of $B\left(D^{+} \rightarrow \omega e^{+} v_{e}\right)$ is determined to be $B\left(D^{+} \rightarrow \omega \mu^{+} v_{\mu}\right) / B\left(D^{+} \rightarrow \omega e^{+} v_{e}\right)=1.05 \pm 0.14$, which is agreement with the SM exectation within $1 \sigma$. 


\section{2 $D_{S}^{+}$Semileptonic decay}

3.2.1 $D_{s}^{+} \rightarrow \eta^{(\prime)} e^{+} v_{e}$

Based on the $3.19 \mathrm{fb}^{-1}$ data set taken at $E_{\mathrm{cm}}=4.178 \mathrm{GeV}$, we measure the absoulte BFs of $D_{S}^{+} \rightarrow \eta e^{+} v_{e}$ and $D_{S}^{+} \rightarrow \eta^{\prime} e^{+} v_{e}$ to be $B\left(D_{s}^{+} \rightarrow \eta e^{+} v_{e}\right)=(2.323 \pm 0063 \pm 0.063) \times 10^{-2}$ and $B\left(D_{s}^{+} \rightarrow \eta^{\prime} e^{+} v_{e}\right)=(0.824 \pm 0073 \pm 0.027) \%$ [15]. From the first measurements of the dynamics of $D_{s}^{+} \rightarrow \eta^{(\prime)} e^{+} v_{e}$ decays, the products of the hadronic form factors $f_{+}^{\eta^{(\prime)}}(0)$ and CKM matrix $\left|V_{c S}\right|$ are determined with different form factor parametrizations. For the two-parameter series expansion, the results are $f_{+}^{\eta}(0)\left|V_{c S}\right|=0.4455 \pm 0.0053 \pm 0.0044$ and $f_{+}^{\eta^{\prime}}(0)\left|V_{c S}\right|=$ $0.477 \pm 0.049 \pm 0.011$. Taking the $\left|V_{c s}\right|$ from the global fit [2], The form factor of $f_{+}^{\eta}(0)$ and $f_{+}^{\eta^{\prime}}(0)$ are determined to be $f_{+}^{\eta}(0)=0.4576 \pm 0.0054 \pm 0.0045$ and $f_{+}^{\eta^{\prime}}(0)=0.490 \pm$ $0.050 \pm 0.011$. In addition, the $\eta-\eta^{\prime}$ mixing angle in the quark flavor basis is determined to be $\phi_{P}=(40.1 \pm 2.1 \pm 0.7)^{\circ}$.

\subsection{2 $D_{s}^{+} \rightarrow K^{0} e^{+} v_{e}$ and $D_{S}^{+} \rightarrow K^{* 0} e^{+} v_{e}$}

We report the measurement of $D_{s}^{+} \rightarrow K^{0} e^{+} v_{e}$ and $D_{s}^{+} \rightarrow K^{* 0} e^{+} v_{e}$ [16]. The absolute BFs are determined to be $B\left(D_{s}^{+} \rightarrow K^{0} e^{+} v_{e}\right)=(3.25 \pm 0.38 \pm 0.16) \times 10^{-3}$ and $B\left(D_{s}^{+} \rightarrow\right.$ $\left.K^{* 0} e^{+} v_{e}\right)=(2.37 \pm 0.26 \pm 0.20) \times 10^{-3}$, with precision significantly improved relative to previous results. In this paper, we obtain the hadronic form-factor parameters for the first time. For $D_{s}^{+} \rightarrow K^{0} e^{+} v_{e}, f_{+}(0)=0.720 \pm 0.084 \pm 0.013$. For $D_{s}^{+} \rightarrow K^{* 0} e^{+} v_{e}, r_{V}=V(0) / A_{1}(0)=$ $1.67 \pm 0.34 \pm 0.16$ and $r_{2}=A_{2}(0) / A_{1}(0)=0.77 \pm 0.28 \pm 0.07$. With the form factor of $D^{+} \rightarrow \pi^{0} e^{+} v_{e}$ [17] and $D^{+} \rightarrow \rho^{0} e^{+} v_{e}$ [18], we calculate the ratio of the form factor of $D_{s}^{+} \rightarrow$ $K^{0} e^{+} v_{e}$ to $D^{+} \rightarrow \pi^{0} e^{+} v_{e}$ and $D_{s}^{+} \rightarrow K^{* 0} e^{+} v_{e}$ to $D^{+} \rightarrow \rho^{0} e^{+} v_{e}$, which are consistent with LQCD predictions and the expectation of $U$-spin $(d \rightarrow s)$ symmetry.

\section{Conclusions}

In summary, BESIII has reported the most precise measurements of the decay constants of the leptonic $D_{(s)}$ decays, the hadronic form factors of semileptonic $D$ decays and CKM matrix elements $\left|V_{c d(s)}\right|$. Meanwhile, LFU tests using these decays has been performed and no evidence of violation is found. Searches for charm semileptonic decays to scalar mesons were performed and our results are in favor of the tetraquark description of $a_{0}(980), f_{0}(500)$ and $f_{0}(980)$. The semileptonic $D$ to axial-vector meson $D^{+} \rightarrow \bar{K}_{1}(1270)^{0} e^{+} v_{e}$ is observed for the first time. With more data coming from BESIII, experimental studies of leptonic and semileptonic $D_{(s)}$ decays will be further improved in the future.

\section{References}

[1] M. Ablikim et al. (BESIII Collaboration), Phys. Rev. Lett. 122, 071082 (2019).

[2] M. Tanabashi et al. (Particle Data Group), Phys. Rev. D 98, 030001 (2018).

[3] A. Bazavov, C. Bernard, N. Brown, C. DeTar, A. X. ElKhadra, E. Gamiz, S. Gottlieb, U. M. Heller, J. Komijani, A. S. Kronfeld, J. Laiho, P. B. Mackenzie, E. T. Neil, J. N. Simone, R. L. Sugar, D. 
Toussaint, and R. S. Van de Water (Fermilab Lattice and MILC Collaborations), Phys. Rev. D 98 074512 (2018).

[4] N. Carrasco, P. Dimopoulos, R. Frezzotti, P. Lami, V. Lubicz, F. Nazzaro, E. Picca, L. Riggio, G. C. Rossi, F. Sanfilippo, S. Simula, and C. Tarantino (ETM Collaboration), Phys. Rev. D 91, 054507.

[5] M. Ablikim et al. (BESIII Collaboration), Phys. Rev. Lett. 123, 211802 (2019).

[6] M. Ablikim et al. (BESIII Collaboration), Phys. Rev. Lett. 122, 011804 (2019).

[7] M. Ablikim et al. (BESIII Collaboration), Phys. Rev. D 92, 072012 (2015).

[8] H. Na, C. T. H. Davies, E. Follana, G. P. Lepage, and J. Shigemitsu (HPQCD Collaboration), Phys. Rev. D 82, 114506 (2010).

[9] M. Ablikim et al. (BESIII Collaboration), Phys. Rev. Lett. 124, 231801 (2020).

[10] M. Ablikim et al. (BESIII Collaboration), Phys. Rev. Lett. 122, 062001 (2019).

[11] M. Ablikim et al. (BESIII Collaboration), Phys. Rev. Lett. 123, 231801 (2019).

[12] H. Y. Cheng and X. W. Kang, Eur. Phys. J. C 77, 587 (2017); 77, 863(E) (2017), and private communication.

[13] S. Momeni and R. Khosravi, J. Phys. G 46, 105006 (2019)

[14] M. Ablikim et al. (BESIII Collaboration), Phys. Rev. D 101, 072005 (2020).

[15] M. Ablikim et al. (BESIII Collaboration), Phys. Rev. Lett. 122, 121801 (2019).

[16] M. Ablikim et al. (BESIII Collaboration), Phys. Rev. Lett. 122, 061801 (2019).

[17] M. Ablikim et al. (BESIII Collaboration), Phys. Rev. D 96, 012002 (2017).

[18] S. Dobbs et al. (CLEO Collaboration), Phys. Rev. Lett. 110, 131802 (2013). 